\title{
Proton pump inhibitors decrease melanogenesis in melanocytes
}

\author{
SEUNG-HWA BAEK ${ }^{1}$ and SANG-HAN LEE ${ }^{1,2}$ \\ Departments of ${ }^{1}$ Food Science and Biotechnology and ${ }^{2}$ Nano-Science and Technology, \\ Kyungpook National University, Daegu 702-701, Republic of Korea
}

Received April 27, 2015; Accepted June 10, 2015

DOI: $10.3892 /$ br.2015.492

\begin{abstract}
Proton pump inhibitors (PPIs) are widely used as inhibitors of gastric juice secretion for treatment of gastroesophageal reflux disease. However, there are no previous studies of the effects on melanogenesis resulting from PPI treatments. Therefore, the aim of the present study was to investigate the effects of PPIs on melanogenesis in melan-a cells derived from immortalized mouse melanocytes. Tyrosinase activity and copper-chelating activity were measured spectrophotometrically. In addition, the melanin content and viability of melan-a cells treated with PPIs were assessed and the mRNA levels of melanogenesis-associated genes were measured by reverse transcription-polymerase chain reaction. Treatment with rabeprazole, but not the other PPIs tested, resulted in strong, dose-dependent inhibition of mushroom tyrosinase (TYR). By contrast, each of the PPIs tested exhibited copper-chelating activity. Treatment of melan-a cells with $100 \mu \mathrm{M}$ concentrations of the PPIs resulted in significantly reduced melanin synthesis and reduced expression of several melanogenesis-associated genes, including $T Y R$, TYR-related protein-1 (TRP-1) and TRP-2, and microphthalmia-associated transcription factor, but did not result in cytotoxic effects. These results suggest that PPIs inhibit melanin biosynthesis in melan-a cells via the downregulation of melanogenesis-associated genes. Furthermore, the findings indicate that PPIs in general could be utilized as skin-whitening agents and/or as biomaterial for treating hyperpigmentation disorders.
\end{abstract}

\section{Introduction}

Proton pump inhibitors (PPIs) are commonly used to treat acid reflux disease and ulcers of the stomach and the duodenum. These compounds include omeprazole, rabeprazole, esomeprazole, lansoprazole and pantoprazole, which are available under a variety of brand names (1). Multiple studies have examined

Correspondence to: Dr Sang-Han Lee, Department of Food Science and Biotechnology, Kyungpook National University, 80 Dahak-ro, Daegu 702-701, Republic of Korea

E-mail: sang@knu.ac.kr

Key words: proton pump inhibitors, tyrosinase, anti-melanogenic effects, melanogenesis-associated genes, melanocyte the cells that line the stomach, regarding the reduction of acid production, which is relatively well-understood (2). Recently, however, it was reported that treatment with PPIs could worsen the symptoms of vitiligo in patients suffering from this skin pigmentation disorder (3). In one study, the investigators demonstrated that PPI treatment could lead to increased destruction of melanocytes in vitiligo patients via the induction of apoptosis (4). Furthermore, one African-American patient receiving PPIs proceeded to widespread vitiligo at the same time as when the daily use of a PPI, $40 \mathrm{mg}$ esomeprazole, began during the 3 years. Following the termination of esomeprazole intake, these patients were able to initiate an extremely slow repigmentation (5). The effects of PPIs on melanogenesis, however, have rarely been recognized.

Melanogenensis is a complex process involving numerous different metabolic signals and a series of enzymes. In human melanocytes, the melanin pigment is derived from hydroxylation of L-tyrosine to L-3,4-dihydroxyphenylalanine (L-DOPA) by tyrosinase (TYR) (EC 1.14.18.1) with two copper ions at the active center (6). Overexpression of the genes involved in pigment formation was shown to result in pigmentation disorders, including lentigo senilis and urticaria pigmentosa, as well as in skin discoloration issues, such as melasma, freckles and chloasma. Thus, TYR is a primary target of screens designed to identify depigmentation agents. In addition to its role in pigmentation disorders, TYR has been revealed to have a role in Parkinson's disease (7) and other neurodegenerative diseases (8). Therefore, the development of anti-melanogenic agents is an important goal for the cosmetic and medicinal industry (9). Melanin biosynthesis is modulated by the expression of microphthalmia-associated transcription factor (MITF), which regulates the transcriptional expression of the TYR, TYR-related protein-1 (TRP-1) and TRP-2 genes in melanocytes (10).

A previous study demonstrated that omeprazole treatment significantly reduced melanogenesis by inhibiting ATP7A gene expression and by enhancing degradation of TYR (11); however, the anti-melanogenic effects of other PPIs are yet to be examined. The objective of the present study was to assess the effects of PPIs on melanogenesis in melanocytes.

\section{Materials and methods}

Materials. Arbutin, L-DOPA, L-tyrosine, $N$-phenylthiourea (PTU), 12-O-tetradecanoylphorbol-13-acetate (TPA), TYR, sodium hydroxide $(\mathrm{NaOH})$, thiazolyl blue tetrazolium bromide 
(MTT), dimethyl sulfoxide (DMSO), rabeprazole, esomeprazole, lansoprazole and pantoprazole were obtained from Sigma-Aldrich (St. Louis, MO, USA). All other reagents and chemicals were of high-grade and are commercially available.

Measurement of mushroom TYR activity. TYR activity was measured as described previously (6). The mushroom TYR (EC 1.14.18.1) reactions were performed in 96-well microplates (SPL Life Sciences Co., Ltd., Pocheon, Gyeonggi, Korea) and consisted of $150 \mu \mathrm{l}$ of $0.1 \mathrm{M}$ phosphate buffer (pH 6.5), $3 \mu \mathrm{l}$ of test sample, $36 \mu \mathrm{l}$ of $1.5 \mathrm{mM}$ L-tyrosine and $7 \mu \mathrm{l}$ of mushroom TYR $[2,100 \mathrm{U} / \mathrm{ml}$ in $0.05 \mathrm{M}$ phosphate buffer, $(\mathrm{pH}$ 6.5)]. The initial absorbance of each mixture and the absorbance after incubation at $37^{\circ} \mathrm{C}$ for $30 \mathrm{~min}$, was measured at $490 \mathrm{~nm}$ using a microplate reader (PerkinElmer, Waltham, MA, USA). The inhibitory activity was subsequently calculated using the following formula: Inhibitory activity $(\%)=[(A-B)-(C-D)] /(A-B) \times 100$; where $A$ refers to the absorbance of the control sample following the reaction, $B$ is the absorbance of the control sample prior to the reaction, $C$ is the absorbance of the treated sample following the reaction and $D$ is the absorbance of the treated sample prior to the reaction.

Measurement of copper-chelating activity. The copper-chelating activity was determined using the pyrocatechol violet (PV) method as described previously, and with slight modifications (12). Samples were mixed with $1 \mathrm{ml}$ of $50 \mathrm{mM}$ sodium acetate buffer $(\mathrm{pH} 6.0)$ and $100 \mu \mathrm{l}$ of $2 \mathrm{mM}$ $\mathrm{CuSO}_{4}$. After $10 \mathrm{~min}$ of incubation at room temperature, $100 \mu \mathrm{l}$ of $2 \mathrm{mM}$ PV was added. Solutions were incubated for $20 \mathrm{~min}$ at room temperature and the absorbance of each sample at $632 \mathrm{~nm}\left(\mathrm{~A}_{632}\right)$ was measured. DMSO and PTU were used as a negative control and as a standard metal chelator, respectively. The copper-chelating activity was calculated using the following formula: Copper-chelating activity $(\%)=[1-(\mathrm{A}-\mathrm{B}) /(\mathrm{C}-\mathrm{D})] \mathrm{x} 100$; where $\mathrm{A}$ is the $\mathrm{A}_{632}$ of the sample + buffer $+\mathrm{CuSO}_{4}+\mathrm{PV}$ mixture, $\mathrm{B}$ is the $\mathrm{A}_{632}$ of the sample + buffer + buffer + PV mixture, $C$ is the $A_{632} \mathrm{~nm}$ of the buffer + buffer $+\mathrm{CuSO}_{4}+\mathrm{PV}$ mixture and D is the $\mathrm{A}_{632} \mathrm{~nm}$ of the buffer + buffer + buffer + PV mixture.

Cell cultures. The melan-a immortalized mouse melanocyte cell line, which was derived from B57BL6 mice (13), was purchased from Bennett et al (13) (St. George's University of London, London, UK). The cells were cultured in RPMI-1640 medium (HyClone, Logan, UT, USA) supplemented with $10 \%$ fetal bovine serum (HyClone), streptomycin-penicillin (100 $\mu \mathrm{g} / \mathrm{ml}$ each; HyClone) and $200 \mathrm{nM}$ TPA (a potent tumor promoter) at $37^{\circ} \mathrm{C}$ in $5 \% \mathrm{CO}_{2}$. Cells were passaged every 3 days up to a maximal passage number of 40 . Confluent monolayers of melanocytes were harvested using a mixture of $0.05 \%$ trypsin and $0.53 \mathrm{mM}$ EDTA (Lonza, Basel, Switzerland).

Cell viability assay. Cell viability was determined using an MTT assay (6). Several concentrations of test samples $(25,50$, 100 and $200 \mu \mathrm{M}$ ) were added to the cells and incubated for $24 \mathrm{~h}$. A total of $100 \mu \mathrm{l}$ of MTT solution [5 mg/ml MTT in phosphate-buffered saline (PBS)] was added to each well and samples were incubated at $37^{\circ} \mathrm{C}$ for $1 \mathrm{~h}$. After removal of the
MTT solution, $1 \mathrm{ml}$ of DMSO was added to each well with vigorous mixing. The absorbance of each well at $470 \mathrm{~nm}$ was determined using a microplate reader (PerkinElmer).

Melanization inhibition assay using melan-a cells. Cells were seeded in 24-well plates (BD Biosciences, Franklin Lakes, NJ, USA) at a density of $1 \times 10^{5}$ cells/well and allowed to attach overnight. The supernatants were subsequently removed and replaced with fresh media containing various concentrations $(25,50,100$ and $200 \mu \mathrm{M})$ of the test compounds. Cells were cultured for $72 \mathrm{~h}$ and further incubated for 1 day. Following washing with PBS, the cells were lysed with $250 \mu \mathrm{l}$ of $1 \mathrm{~N}$ $\mathrm{NaOH}$ and transferred to 96 -well plates. The melanin content was estimated by measuring the absorbance at $405 \mathrm{~nm}$ using a microplate reader (PerkinElmer). Arbutin was used as a positive control (10).

Reverse transcription-polymerase chain reaction (RT-PCR) analysis of $m R N A$ expression. Total RNA was extracted from melan-a cells using TRIzol reagent (Invitrogen Life Technologies, Waltham, MA, USA), according to the manufacturer's instructions, with slight modification (14). Total RNA $(2 \mu \mathrm{g})$ was reverse transcribed with RT (MP Biomedicals, Santa Ana, CA, USA) and oligo (dT) primers. cDNA was amplified using a PCR Thermal Cycler Dice TP600 (Takara Bio, Inc., Shiga, Japan) and oligonucleotide primers specific for mouse transcripts (6). The resulting PCR products were visualized by ethidium bromide staining following agarose gel electrophoresis.

Statistical analysis. Data are expressed as the means \pm standard deviation. Statistical significance was determined by the Student's t-test for independent means using Microsoft Excel software (Microsoft Corporation, Redmond, WA, USA). P<0.05 was considered to indicate a statistically significant difference.

\section{Results}

Effect of PPIs on TYR and copper-chelating activity. The effect of PPIs on the activity of TYR, a crucial enzyme for melanin synthesis, was examined. As depicted in Fig. 1, rabeprazole treatment resulted in significant, dose-dependent inhibition of TYR activity and the $50 \%$ inhibitory concentration $\left(\mathrm{IC}_{50}\right)$ of this compound was $110.1 \mu \mathrm{M}$. The $\mathrm{IC}_{50}$ of the arbutin positive control was $\sim 212.3 \mu \mathrm{M}$ (Fig. 1A). By contrast, the PPIs esomeprazole, lansoprazole and pantoprazole exerted no inhibitory effect on TYR activity.

To understand the role of metal chelation in PPI TYR inhibition, the copper-chelating activities of each PPI were examined. In general, each of the PPIs tested exhibited strong copper-chelation activities. The copper chelation values of the PPIs were higher compared with that of the PTU positive control (Fig. 1B). While esomeprazole, lansoprazole and pantoprazole did not affect TYR activity, these compounds did exhibit copper-chelating activity. Conversely, rabeprazole exhibited TYR inhibitory and copper-chelating activity, indicating that this compound may inhibit TYR by chelating copper.

Effects of PPIs on cell viability and melanin content in melan-a cells. To examine the effect of PPIs on melanin biosynthesis, 
A

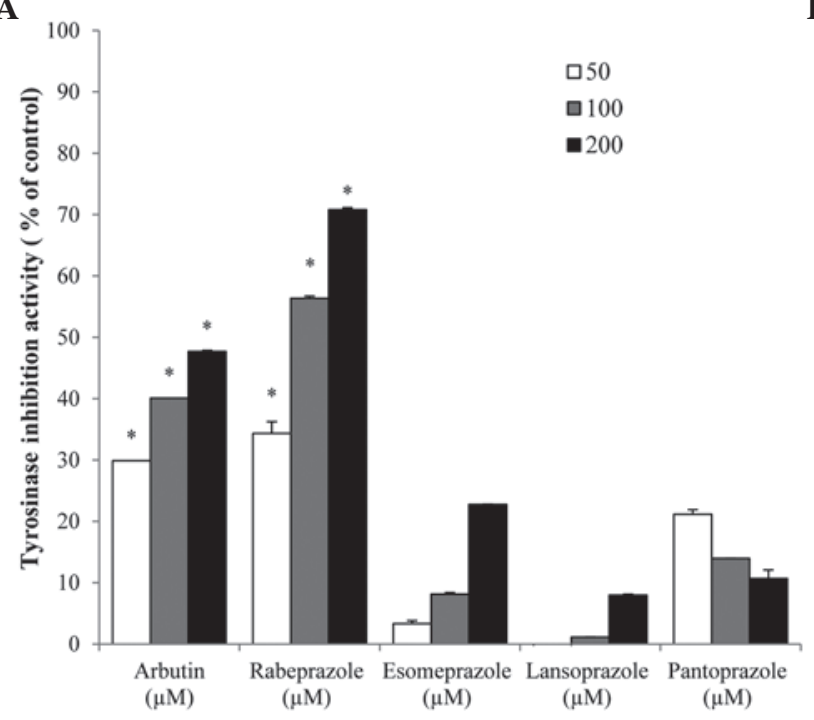

B

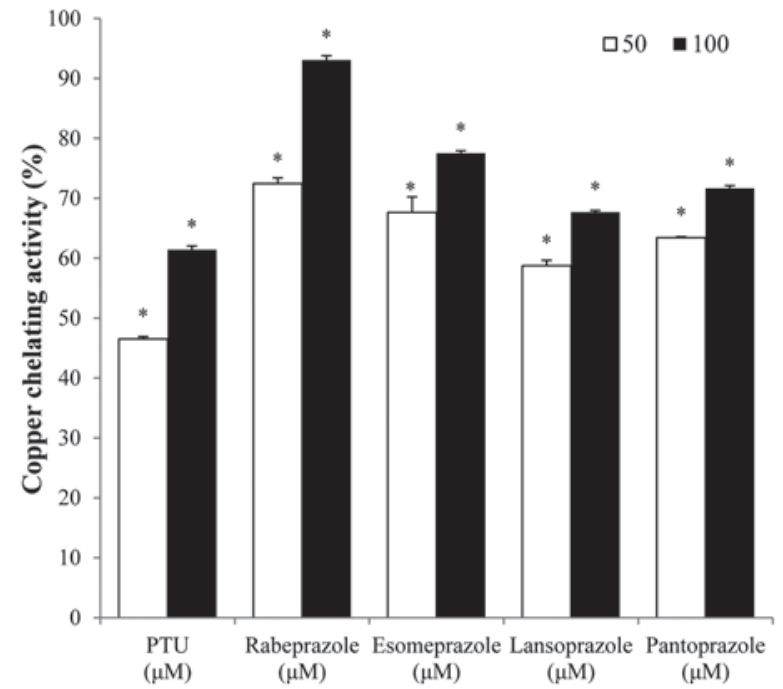

Figure 1. Effects of proton pump inhibitors on mushroom tyrosinase activity and copper chelation. Tyrosinase was preincubated with each of the test substances at $25^{\circ} \mathrm{C}$ for $5 \mathrm{~min}$ prior to incubation with L-tyrosine for $30 \mathrm{~min}$. (A) The absorbance of each solution was measured at $490 \mathrm{~nm}$. The copper-chelating activity was determined using the pyrocatechol violet (PV) method. Samples were preincubated with $50 \mathrm{mM}$ sodium acetate buffer (pH 6.0 ) and $2 \mathrm{mM} \mathrm{CuSO}$ at room temperature for $10 \mathrm{~min}$ prior to incubation with $2 \mathrm{mM}$ PV for $20 \mathrm{~min}$. (B) The absorbance of each sample at $632 \mathrm{~nm}$ was measured. Each experiment was performed in triplicate, and the data shown represent mean \pm standard deviation. Student's t-test was utilized to compare the results and $\mathrm{P}<0.05$ was considered to indicate a statistically significant difference to each control group. PTU, $N$-phenylthiourea.

A

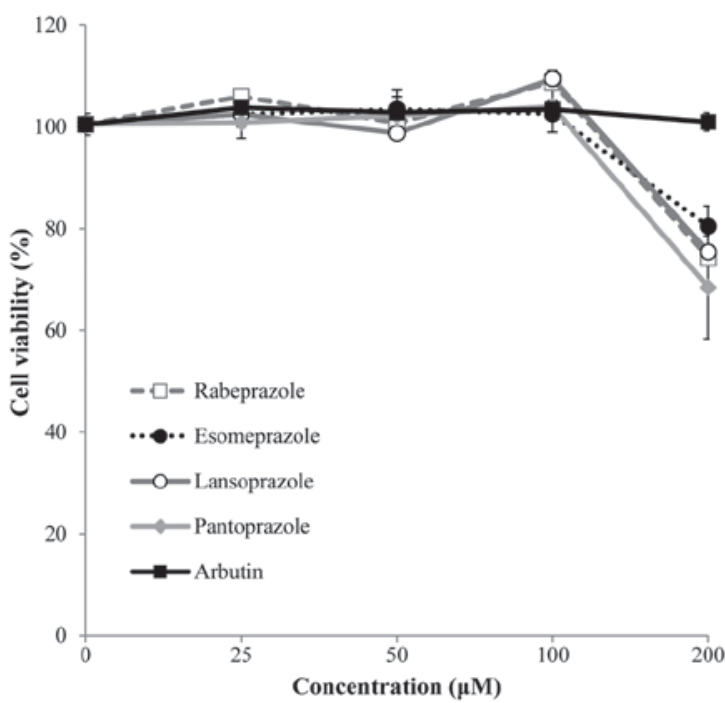

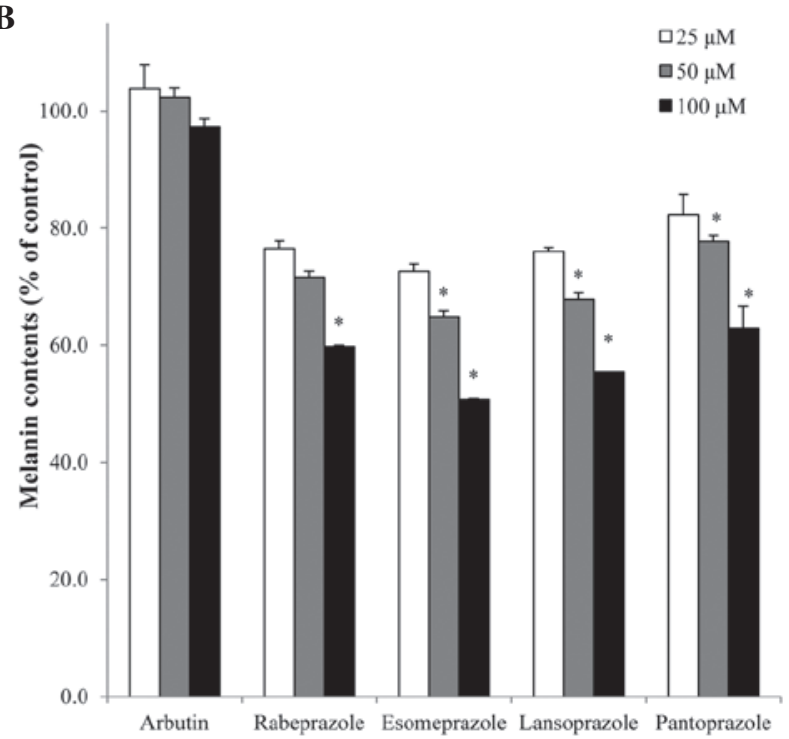

Figure 2. Effects of proton pump inhibitors (PPIs) on melanogenesis in melan-a cells. Melan-a cells (1x10 cells/ml) were cultured for $24 \mathrm{~h}$ and the medium was replaced with fresh medium containing various concentrations $(25,50,100$ and $200 \mu \mathrm{M})$ of either a PPI or arbutin. Cells were subsequently incubated for 3 days and (A) cell viability was measured using thiazolyl blue tetrazolium bromide assay. The cells were collected and lysed with $1 \mathrm{~N}$ sodium hydroxide and (B) the melanin content of each sample was estimated by measuring the absorbance at $405 \mathrm{~nm}$. Each treatment was performed in triplicate and the data shown represent mean \pm standard deviation. Student's t-test was utilized to compare the results and ${ }^{*} \mathrm{P}<0.05$ was considered to indicate a statistically significant difference to the control group.

melan-a cells were treated with various concentrations $(25,50$, 100 and $200 \mu \mathrm{M}$ ) of PPIs, and melanin content and cell viability were subsequently assessed. No cytotoxicity was observed in the melanocytes treated with PPIs or arbutin at concentrations of $\leq 100 \mu \mathrm{M}$ (Fig. 2A). Cytotoxicity in melan-a populations treated with $200 \mu \mathrm{M}$ of rabeprazole, esomeprazole, lansoprazole and pantoprazole was observed as $\sim 25.8 \pm 6.9,19.5 \pm 3.9$, $24.6 \pm 5.8$ and $31.6 \pm 10.1 \%$, respectively.

At a concentration of $100 \mu \mathrm{M}$, PPI treatment resulted in significant decreases in melanin content without inducing cell death. Specifically, treatment with rabeprazole, esomeprazole, lansoprazole and pantoprazole resulted in $40.2 \pm 0.2,49.3 \pm 0.2$, $44.6 \pm 0.0$ and $37.1 \pm 3.8 \%$ decreases in melanin content, compared with that of the control cell population, respectively (Fig. 2B). These results indicate that treatment with PPIs decreased melanin synthesis without influencing melanocyte viability in vitro.

Effects of PPIs on expression of melanogenesis-associated genes in melan-a cells. The influence of PPIs on the mRNA 


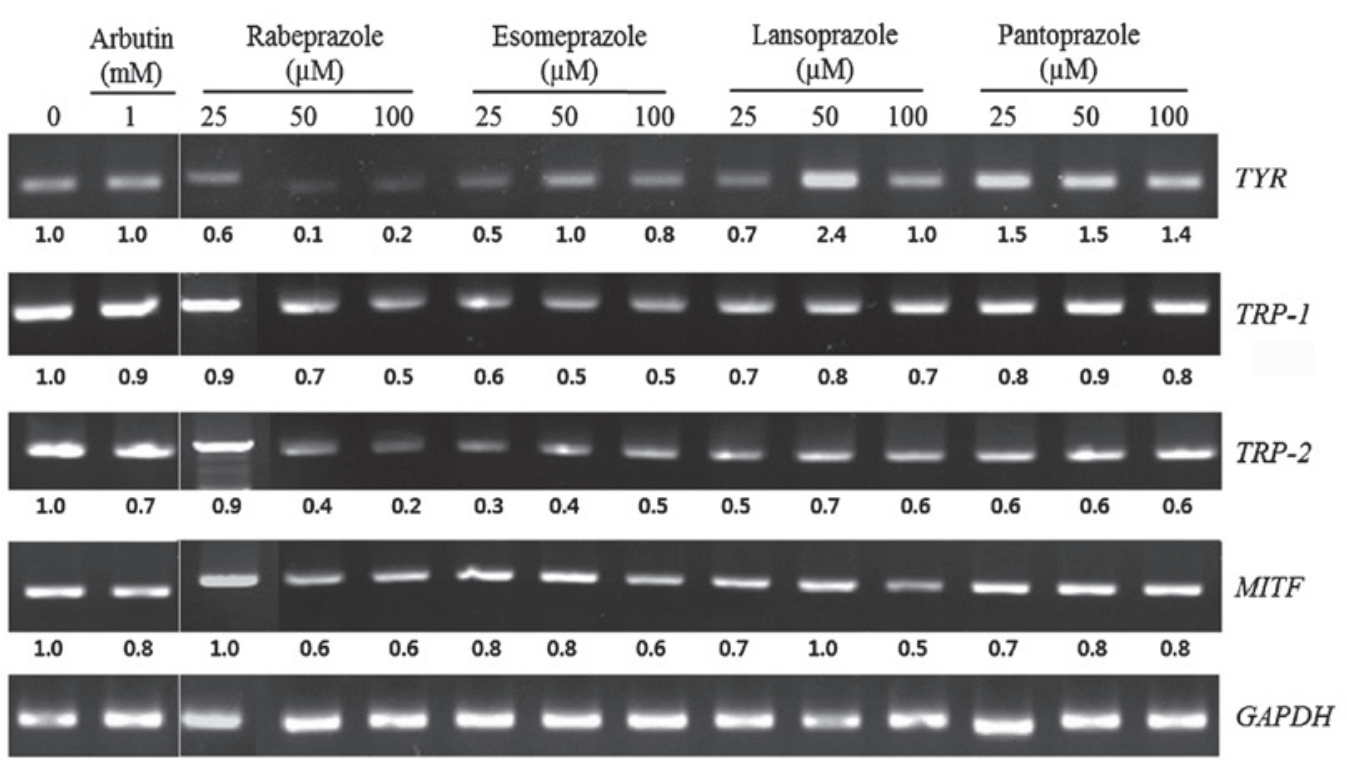

Figure 3. Effects of proton pump inhibitors (PPIs) on melanogenesis-associated gene expression in melan-a cells. Melan-a cells (1x10 5 cells/ml) were cultured for $24 \mathrm{~h}$. The medium was subsequently replaced with fresh medium containing various concentrations (25, 50 and $100 \mu \mathrm{M})$ of either a PPI compound or arbutin and cells were incubated for 1 day. The cells were collected and RNA was harvested using TRIzol reagent. Following quantifying RNA levels, mRNA expression levels were analyzed by reverse transcription-polymerase chain reaction. Band intensities were normalized to that of glyceraldehyde 3-phosphate dehydrogenase (GAPDH) using image J software. TYR, tyrosinase; TRP-1, tyrosinase-related-1.

levels of the TYR, TRP-1, TRP-2 and MITF genes was examined using RT-PCR. Treatment with $100 \mu \mathrm{M}$ rabeprazole suppressed the expression of the TYR, TRP-1, TRP-2 and MITF genes by $77.1,48.3,76.2$ and $37.1 \%$, respectively. Esomeprazole $(100 \mu \mathrm{M})$ decreased the expression of these genes by $23.1,47.9,53.9$ and $30.7 \%$, respectively, and $100 \mu \mathrm{M}$ lansoprazole inhibited the expression of the TRP-1,TRP-2 and $M I T F$ by $25.9,44.9$ and $52.8 \%$, respectively. Treatment with $100 \mu \mathrm{M}$ pantoprazole suppressed the mRNA expression of the TRP-1, TRP-2 and MITF genes by 22.3, 37.9 and $15.7 \%$, respectively (Fig. 3). Taken together, these results demonstrate that rabeprazole treatment affected mRNA expression of each of the melanogenesis-associated genes.

\section{Discussion}

The present study demonstrated that PPIs act as inhibitors of melanogenesis in melanocytes in vitro. Melanin biosynthesis begins with the hydroxylation of tyrosine to L-DOPA by TYR. In the absence of thiols, the second enzyme, TRP-2, enables a rapid conversion of dopaquinone to dopachrome, and subsequently to 5,6-dihydroxyindole (DHI) or indole 5,6-quinone 2-carboxylic acid (DHICA). TRP-1 catalyzes the oxidation of DHICA to produce eumelanins (15). The active site of TYR contains a binuclear copper center, which catalyzes the hydroxylation of phenols to catechols and the oxidation of catechols to quinones (16). Rabeprazole was the only PPI tested that exhibited mushroom TYR inhibitory activity and copper-chelating activity (Fig. 1). Each of the PPIs tested decreased the melanin content of melan-a cells without inducing cytotoxicity (Fig. 2). Notably, while treatment with esomeprazole, lansoprazole and pantoprazole had no effect on TYR inhibitory activity, these compounds exhibited copper-chelating activity and mediated decreases in melanin formation in melanocytes. These data suggest that esomeprazole, lansoprazole and pantoprazole inhibit melanogenesis via their copper-chelating activity and not via effects on TYR activity.

In mammalian melanocytes, melanin biosynthesis is directly modulated by 3 melanocyte-specific enzymes: TYR, TRP-1 and TRP-2. These melanocyte-specific enzymes are directly regulated by MITF, which is known to be the integral transcription factor for the regulation of melanocyte differentiation and pigmentation (17). Therefore, the mRNA levels of TYR, TRP-1, TRP-2 and MITF were measured following PPI treatment. Rabeprazole strongly suppressed the expression of each of the melanogenesis-associated genes, indicating that this compound transcriptionally regulates $T Y R, T R P-1$ and $T R P-2$ expression through $M I T F$ during melanin biosynthesis. Treatment with lansoprazole also resulted in decreased MITF mRNA levels; however, these compounds had no effect on $T Y R$ expression levels. Therefore, esomeprazole, lansoprazole and pantoprazole were predicted to inhibit melanin formation through some other mechanism. Melanogenesis is modulated by various stimuli, including ultraviolet irradiation, hormone treatment and genetic mutations. Stimulation of the melanocortin 1 receptor (also known as melanocyte-stimulating hormone receptor) activates adenyl cyclase through $\mathrm{G}$ protein signaling, thereby increasing cyclic adenosine monophosphate production, which activates the expression of MITF (6). It has also been reported that stem cell factor ( $\mathrm{SCF}) / \mathrm{c}-\mathrm{kit}$ signaling is associated with the survival, proliferation and differentiation of melanocytes (18). In the epidermis, SCF produced by keratinocytes binds to the receptor on melanocytes and induces the activation of the melanogenesis signaling cascade (19). Thus, further studies are necessary to examine the effects of PPIs on other melanogenic processes.

Previous reports have indicated that PPIs induce depigmentation in patients with vitiligo. In one study, patients developed vitiligo following the use of oral PPIs for treatment of occurred gastritis (20). Additionally, Schallreuter and Rokos (5) identified that the progress of repigmentation was much slower in patients 
who had received PPI treatment compared with those who had previously not been exposed to these compounds. PPIs are widely used to treat stomach and intestinal diseases. PPIs inhibit gastric acid secretion by blocking the gastric $\mathrm{H}^{+} / \mathrm{K}^{+}$-ATPase that is necessary for the final step of this process (21). Melanocytes also express an $\mathrm{H}^{+} / \mathrm{K}^{+}$-ATPase at the cytoplasmic membrane and melanosomes contain a vacuolar type $\mathrm{H}^{+}$-ATPase (4). Matsui et al (11) demonstrated that omeprazole reduces melanogenesis by inhibiting ATP7A expression and by enhancing degradation of TYR. The present data are consistent with recent findings that pantoprazole decreased melanin content and TYR activity in B16 melanoma cells. However, the actions of rabeprazole, esomeprazole and lansoprazole were not delineated. Nevertheless, the study provides novel information regarding the inhibitory effects of PPIs on melanogenesis.

\section{Acknowledgements}

The present study was supported by the Basic Science Research Program through the National Research Foundation of Korea (NRF) funded by the Ministry of Science, ICT and Future Planning (grant no. NRF-2014R1A2A2A01006882).

\section{References}

1. Jain KS, Shah AK, Bariwal J, Shelke SM, Kale AP, Jagtap JR and Bhosale AV: Recent advances in proton pump inhibitors and management of acid-peptic disorders. Bioorg Med Chem 15: 1181-1205, 2007

2. Rew JS: Clinical use of proton pump inhibitors in gastrointestinal diseases. Korean J Gastroenterol 47: 181-190, 2006 (In Korean).

3. Shin JM, Lee JY, Lee DY, Yoon TY, Lee JC, Lim EH, Sohn KC, Lee YH, Im M, Seo YJ, et al: Proton pump inhibitors as a possible cause of vitiligo: An in vivo and in vitro study. J Eur Acad Dermatol Venereol 28: 1475-1479, 2014.

4. Namazi MR: Proton pump inhibitors may trigger vitiligo by rendering melanocytes prone to apoptosis. Br J Dermatol 158 : 844-845, 2008

5. Schallreuter KU and Rokos H: From the bench to the bedside: Proton pump inhibitors can worsen vitiligo. Br J Dermatol 156: 1371-1373, 2007.

6. Baek SH, Ahn JW, Nam SH, Yoon CS, Shin JC and Lee SH: S-(-)-10,11-dihydroxyfarnesoic acid methyl ester inhibits melanin synthesis in murine melanocyte cells. Int J Mol Sci 15 12750-12763, 2014.
7. Pan T, Li X and Jankovic J: The association between Parkinson's disease and melanoma. Int J Cancer 128: 2251-2260, 2011.

8. Pan T, Zhu J, Hwu WJ and Jankovic J: The role of alpha-synuclein in melanin synthesis in melanoma and dopaminergic neuronal cells. PLoS One 7: e45183, 2012.

9. Parvez S, Kang M, Chung HS and Bae H: Naturally occurring tyrosinase inhibitors: Mechanism and applications in skin health, cosmetics and agriculture industries. Phytother Res 21: 805-816, 2007.

10. Kim JH, Baek SH, Kim DH, Choi TY, Yoon TJ, Hwang JS, Kim MR, Kwon HJ and Lee CH: Downregulation of melanin synthesis by haginin $\mathrm{A}$ and its application to in vivo lightening model. J Invest Dermatol 128: 1227-1235, 2008.

11. Matsui MS, Petris MJ, Niki Y, Karaman-Jurukovska N, Muizzuddin N, Ichihashi M and Yarosh DB: Omeprazole, a gastric proton pump inhibitor, inhibits melanogenesis by blocking ATP7A trafficking. J Invest Dermatol 135: 834-841, 2015.

12. Rao F, Yuting Z, Yiran G and Fang C: Antioxidant and tyrosinase inhibition activities of the ethanol-insoluble fraction of water extract of Sapium sebiferum (L.) Roxb. leaves. South Afr J Bot 93: 98-104, 2014.

13. Bennett DC, Cooper PJ and Hart IR: A line of non-tumorigenic mouse melanocytes, syngeneic with the B16 melanoma and requiring a tumour promoter for growth. Int J Cancer 39: 414-418, 1987.

14. Heo JC and Lee SH: Alleviation of asthma-related symptoms by a derivative of L-allo threonine. Int J Mol Med 31: 881-887, 2013.

15. Chao HC, Najjaa H, Villareal MO, Ksouri R, Han J, Neffati M and Isoda H: Arthrophytum scoparium inhibits melanogenesis through the down-regulation of tyrosinase and melanogenic gene expressions in B16 melanoma cells. Exp Dermatol 22: 131-136, 2013.

16. Beberok A, Wrześniok D, Otręba M and Buszman E: Impact of sparfloxacin on melanogenesis and antioxidant defense system in normal human melanocytes HEMa-LP - An in vitro study. Pharmacol Rep 67: 38-43, 2015.

17. Tuerxuntayi A, Liu YQ, Tulake A, Kabas M, Eblimit A and Aisa HA: Kaliziri extract upregulates tyrosinase, TRP-1, TRP-2 and MITF expression in murine B16 melanoma cells. BMC Complement Altern Med 14: 166-175, 2014.

18. Hachiya A, Kobayashi A, Ohuchi A, Takema Y and Imokawa G: The paracrine role of stem cell factor/c-kit signaling in the activation of human melanocytes in ultraviolet-B-induced pigmentation. J Invest Dermatol 116: 578-586, 2001.

19. Shin SH and Lee YM: Glyceollins, a novel class of soybean phytoalexins, inhibit SCF-induced melanogenesis through attenuation of SCF/c-kit downstream signaling pathways. Exp Mol Med 45: e17, 2013.

20. Holla AP, Kumar R, Parsad D and Kanwar A: Proton pump inhibitor induced depigmentation in vitiligo. J Cutan Aesthet Surg 4: 46-47, 2011.

21. Shin JM and Sachs G: Pharmacology of proton pump inhibitors. Curr Gastroenterol Rep 10: 528-534, 2008. 